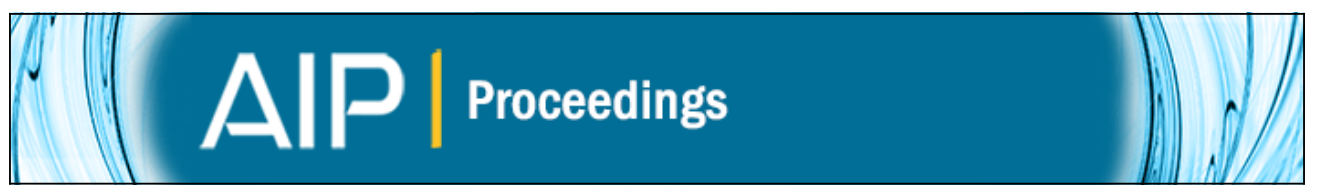

\title{
Creating White Dwarf Photospheres in the Laboratory
}

Ross E. Falcon, G. A. Rochau, J. E. Bailey, J. L. Ellis, M. H. Montgomery, D. E. Winget, Matthew R. Gomez, and R. J. Leeper

Citation: AIP Conference Proceedings 1273, 436 (2010); doi: 10.1063/1.3527858

View online: http://dx.doi.org/10.1063/1.3527858

View Table of Contents:

http://scitation.aip.org/content/aip/proceeding/aipcp/1273?ver=pdfcov

Published by the AIP Publishing

Articles you may be interested in

On the Origin of Metals in Some Hot White Dwarf Photospheres

AIP Conf. Proc. 1331, 289 (2011); 10.1063/1.3556213

Abundance Analysis of DAZ White Dwarfs

AIP Conf. Proc. 1331, 238 (2011); 10.1063/1.3556206

On the origin of metals in some hot white dwarf photospheres

AIP Conf. Proc. 1273, 473 (2010); 10.1063/1.3527867

Ultracool Companions to White Dwarfs

AIP Conf. Proc. 1273, 384 (2010); 10.1063/1.3527847

White Dwarf and Pre-White Dwarf Pulsations

AIP Conf. Proc. 1170, 605 (2009); 10.1063/1.3246571 


\title{
Creating White Dwarf Photospheres in the Laboratory
}

\author{
Ross E. Falcon*, G. A. Rochau ${ }^{\dagger}$, J. E. Bailey ${ }^{\dagger}$, J. L. Ellis*, M. H. \\ Montgomery*, D. E. Winget*, Matthew R. Gomez** and R. J. Leeper ${ }^{\dagger}$ \\ *Department of Astronomy and McDonald Observatory, University of Texas, Austin, TX 78712, \\ USA \\ ${ }^{\dagger}$ Sandia National Laboratories, Albuquerque, New Mexico 87185-1196, USA \\ ** Plasma, Pulsed Power, and Microwave Laboratory, Department of Nuclear Engineering and \\ Radiological Sciences, University of Michigan, Ann Arbor, Michigan 48109-2104, USA
}

\begin{abstract}
We present a preliminary report from the laboratory astrophysics experiments to create macroscopic $\left(\sim 19 \mathrm{~cm}^{3}\right)$ hydrogen-plasmas with white dwarf (WD) photospheric conditions (i.e., temperature, electron density). These experiments, performed at the Z Pulsed Power Facility at Sandia National Laboratories, will serve as benchmarks for fundamental atomic line profile measurements in emission and absorption; they are targeted to address the discrepancy between theory and observation of WD photospheres - cooler photospheres in particular.
\end{abstract}

Keywords: white dwarfs, methods: laboratory, techniques: spectroscopic

PACS: $97.20 . R p, 52.72 .+v, 52.50 .-b$

\section{MOTIVATION}

The most important tool for determining physical properties of white dwarfs (WDs) and assembling them in large numbers is the spectroscopic fitting of absorption lines from observed spectra with those of synthetic spectra from atmosphere models. This technique [e.g., 1] yields surface gravities, effective temperatures, atmospheric compositions, magnetic field strengths and other quantities that provide constraints for asteroseismological studies [e.g., 2] and form the basis for higher level investigations, such as those used for initial-final mass relation studies [e.g., 3] and cosmochronology [4].

Put another way, the conclusions of numerous investigations depend upon the accuracy of spectroscopic fitting. It is well known in the field, however, that the spectroscopic technique is highly problematic for cool $\left(T_{\text {eff }} \lesssim 12,000 \mathrm{~K}\right)$ DA WDs [5, 6], for which it gives systematically higher mass determinations. Photometric [7, 8] and gravitational redshift [9] studies show this mass increase to be unphysical.

DBs suffer from a similar apparent mass increase at $T_{\text {eff }} \lesssim 16,000 \mathrm{~K}$ [7] as well as a highly uncertain location of the DBV instability strip [10,11]. For the study of hot DQs [e.g., 12], spectroscopy is utterly uncalibrated and cannot provide reliable determinations of atmospheric conditions. The same is true for cool DQs, whose $\mathrm{C}_{2}$ Swan bands are not adequately reproduced by models [13].

Meanwhile, outside the WD community and even outside a large portion of the astrophysical community, Sandia National Laboratories has developed the Z Pulsed Power Facility [14] - a machine capable of performing high energy density science experi- 
ments at plasma conditions relevant to all the aforementioned astrophysical problems.

We are currently running experiments at the $\mathrm{Z}$ facility to create WD photospheres in the laboratory. By observing these plasmas spectroscopically and using independent diagnostics to ascertain the plasma conditions (i.e., temperature, electron density), we aim to provide measurements of line profiles that will (1) serve as benchmarks for fundamental atomic physics, and (2) be used to calibrate WD atmosphere models and hence the spectroscopic technique for WD astronomy.

\section{EXPERIMENTAL PLATFORM}

Our experiment uses the X-ray beam line from the opacity experiments of Bailey et al. [15] to uniformly heat a macroscopic $\left(\sim 19 \mathrm{~cm}^{3}\right)$ cell filled with $\mathrm{H}_{2}$ gas. Bailey et al. [15] reach electron temperatures above $150 \mathrm{eV}$ and electron densities near $10^{22} \mathrm{~cm}^{-3}$ in iron-plasmas with the goal of testing the physics of opacity models used for solar interior radiation transport (see also Bailey et al. [16]). The photospheres of DAs do not require such extreme conditions. By placing our gas cell $\sim 35 \mathrm{~cm}$ away from the central experiment, the X-ray flux radially diffuses to a density suitable for our purposes (see Sanford [17] for a description of the radiation environment). It should be noted that ours is not the only astrophysics experiment making use of an X-ray beam line. Hall et al. [18] are investigating the atomic-kinetic and radiative characteristics of photoionized plasmas relevant to such environments as active galactic nuclei and X-ray binaries. All these experiments - Bailey et al. [15], Hall et al. [18] and ours - are utilizing the unique capabilities of the $\mathrm{Z}$ facility and are being performed simultaneously!

The gas cell is $6 \mathrm{~cm}$ long and $2 \mathrm{~cm}$ in diameter. A $1.5 \mu \mathrm{m}$ Mylar window that is stretched across the length of the cell faces the X-ray source. After transmitting through the Mylar, the incident X-ray photons are in the $\sim 100-1000 \mathrm{eV}$ range. This volume of $\mathrm{H}_{2}$ gas is transparent to these $\mathrm{X}$-rays at room temperature, so the inner walls of the cell are lined with gold, which is relatively efficient at absorbing photons of this energy. The gold re-emits lower energy photons, which heat the $\mathrm{H}_{2}$ gas to $\sim 1 \mathrm{eV}$, dissociating and partially ionizing it.

We use a streaked spectrometer system to measure the emission from the hydrogenplasma along the line of sight that runs the length of the cell (Figure 1). A fused silica step index multimode fiber with a core diameter of $200 \mu \mathrm{m}$ collects and delivers light from the gas cell to a $1 \mathrm{~m}$ focal length Czerny-Turner spectrometer (S.I. McPherson, Inc.) housed in a room isolated from the $\mathrm{Z}$ machine. The spectrometer uses a $300 \mathrm{l} / \mathrm{mm}$ grating which gives $\sim$ few $\AA$ spectral resolution. A streak camera with micro-channel plate intensifier placed at the exit of the spectrometer outputs to Kodak TMAX 400 film.

\section{PLASMA CONDITIONS}

The ultimate goal of our experiments is to measure line shapes observed in the spectra of plasmas with independently determined conditions. In the preliminary stages of our project, however, we use the spectra to estimate the plasma conditions for the purpose of confirming proof-of-concept for the experimental setup. 


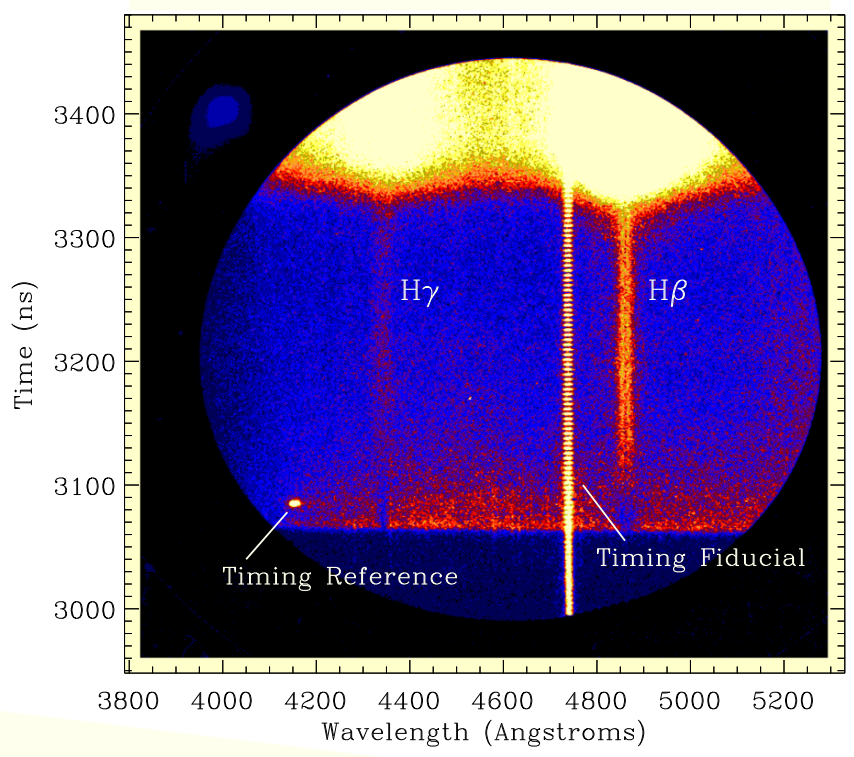

FIGURE 1. Streaked spectrum of H-plasma showing the $\mathrm{H} \beta$ and $\mathrm{H} \gamma$ emission lines. Notice the plasma reaches equilibrium quickly $(\sim 50 \mathrm{~ns})$ and remains stable for $\sim 200 \mathrm{~ns}$.

The gas fill pressure inside the cell is $\sim 15.0$ Torr (total atom density of $\sim 10^{18} \mathrm{~cm}^{-3}$ ). By comparing the observed $\mathrm{H} \beta$ line shape (not shown) to that of Wiese et al. [19], we estimate an electron density of $\sim 8 \times 10^{16} \mathrm{~cm}^{-3}$, which implies an ionization fraction of $\sim 0.08$. Assuming LTE, the temperature can be calculated from the Saha equation [e.g., 20] which yields $\sim 1 \mathrm{eV}$ ( or $\sim 12,000 \mathrm{~K})$.

\section{OUTLOOK}

We continue to make improvements to the gas cell and experimental design. Modifications have already resulted in significant decreases (or elimination) of scattered light within the gas cell as well as an increased lifetime of the plasma in its stable phase.

We are currently working to implement absolute intensity calibrations and independent diagnostics for the plasma conditions.

\section{ACKNOWLEDGMENTS}

This work was performed at Sandia National Laboratories. We thank the Z dynamic hohlraum, accelerator, diagnostics, materials processing, target fabrication, and wire array teams, without which we cannot run our experiments. Sandia is a multiprogram 
laboratory operated by Sandia Corporation, a Lockheed Martin Company, for the United States Department of Energy under Contract No. DE-AC04-94AL85000.

\section{REFERENCES}

1. P. Bergeron, R. A. Saffer, and J. Liebert, ApJ, 394, 228-247 (1992).

2. S. E. Thompson, M. H. Montgomery, T. von Hippel, A. Nitta, J. Dalessio, J. Provencal, W. Strickland, J. A. Holtzman, A. Mukadam, D. Sullivan, T. Nagel, D. Koziel-Wierzbowska, T. Kundera, S. Zola, M. Winiarski, M. Drozdz, E. Kuligowska, W. Ogloza, Z. Bognár, G. Handler, A. Kanaan, T. Ribeira, R. Rosen, D. Reichart, J. Haislip, B. N. Barlow, B. H. Dunlap, K. Ivarsen, A. LaCluyze, and F. Mullally, ApJ, 714, 296-308 (2010).

3. K. A. Williams, M. Bolte, and D. Koester, ApJ, 693, 355-369 (2009).

4. D. E. Winget, C. J. Hansen, J. Liebert, H. M. van Horn, G. Fontaine, R. E. Nather, S. O. Kepler, and D. Q. Lamb, ApJL, 315, L77-L81 (1987).

5. P. Bergeron, A. Gianninas, and S. Boudreault, "The Mass Distribution of White Dwarfs: An Unwavering Obsession," in 15th European Workshop on White Dwarfs, edited by R. Napiwotzki, and M. R. Burleigh, 2007, vol. 372 of Astronomical Society of the Pacific Conference Series, 29.

6. D. Koester, S. O. Kepler, S. J. Kleinman, and A. Nitta, Journal of Physics Conference Series, 172, 012006 (2009).

7. S. O. Kepler, S. J. Kleinman, A. Nitta, D. Koester, B. G. Castanheira, O. Giovannini, A. F. M. Costa, and L. Althaus, MNRAS, 375, 1315-1324 (2007).

8. A. Engelbrecht, and D. Koester, "Photometric Calibration and DAs in the SDSS," in 15th European Workshop on White Dwarfs, edited by R. Napiwotzki \& M. R. Burleigh, 2007, vol. 372 of Astronomical Society of the Pacific Conference Series, 289.

9. R. E. Falcon, D. E. Winget, M. H. Montgomery, and K. A. Williams, ApJ, 712, 585-595 (2010).

10. A. Beauchamp, F. Wesemael, P. Bergeron, G. Fontaine, R. A. Saffer, J. Liebert, and P. Brassard, ApJ, 516, 887-891 (1999).

11. A. H. Córsico, L. G. Althaus, M. M. Miller Bertolami, and E. García-Berro, Journal of Physics Conference Series, 172, 012075 (2009).

12. P. Dufour, J. Liebert, B. Swift, G. Fontaine, and T. Sukhbold, Journal of Physics Conference Series, 172, $012012(2009)$

13. P. M. Kowalski, A\&A, 519, L8+ (2010).

14. W. A. Stygar, M. E. Cuneo, D. I. Headley, H. C. Ives, R. J. Leeper, M. G. Mazarakis, C. L. Olson, J. L. Porter, T. C. Wagoner, and J. R. Woodworth, Physical Review Special Topics Accelerators and Beams, 10, 030401 (2007).

15. J. E. Bailey, G. A. Rochau, C. A. Iglesias, J. Abdallah, Jr., J. J. Macfarlane, I. Golovkin, P. Wang, R. C. Mancini, P. W. Lake, T. C. Moore, M. Bump, O. Garcia, and S. Mazevet, Physical Review Letters, 99, 265002 (2007).

16. J. E. Bailey, G. A. Rochau, R. C. Mancini, C. A. Iglesias, J. J. Macfarlane, I. E. Golovkin, C. Blancard, P. Cosse, and G. Faussurier, Physics of Plasmas, 16, 058101 (2009).

17. T. W. L. Sanford, IEEE Transactions on Plasma Science, 36, 22-36 (2008).

18. I. M. Hall, T. Durmaz, R. C. Mancini, J. E. Bailey, G. A. Rochau, M. J. Rosenberg, D. H. Cohen, I. E. Golovkin, J. J. Macfarlane, M. E. Sherril, J. Abdallah, R. F. Heeter, M. E. Foord, S. H. Glenzer, and H. A. Scott, Ap\&SS, 322, 117-121 (2009).

19. W. L. Wiese, D. E. Kelleher, and D. R. Paquette, Physical Review A, 6, 1132-1153 (1972).

20. C. J. Hansen, S. D. Kawaler, and V. Trimble, Stellar interiors : physical principles, structure, and evolution, 2004. 